\title{
Role of interlayer coupling for the power factor of $\mathrm{CuSbS}_{2}$ and $\mathrm{CuSbSe}_{2}$
}

\author{
Najebah M. Alsaleh, Nirpendra Singh, and Udo Schwingenschlögl* \\ Physical Science and Engineering Division (PSE), King Abdullah University of Science and Technology (KAUST), \\ Thuwal 23955-6900, Saudi Arabia
}

(Received 16 June 2016; published 26 September 2016)

\begin{abstract}
The electronic and transport properties of bulk and monolayer $\mathrm{CuSbS}_{2}$ and $\mathrm{CuSbSe}_{2}$ are determined by using density functional theory and semiclassical Boltzmann transport theory, in order to investigate the role of interlayer coupling for the thermoelectric properties. The calculated band gaps of the bulk compounds are in agreement with experiments and significantly higher than those of the monolayers, which thus show lower Seebeck coefficients. Since also the electrical conductivity is lower, the monolayers are characterized by lower power factors. Therefore, interlayer coupling is found to be essential for the excellent thermoelectric response of $\mathrm{CuSbS}_{2}$ and $\mathrm{CuSbSe}_{2}$, even though it is weak.
\end{abstract}

DOI: 10.1103/PhysRevB.94.125440

\section{INTRODUCTION}

$\mathrm{CuSbS}_{2}$ and $\mathrm{CuSbSe}_{2}$ are currently being discussed as promising materials for solar cell applications due to their high absorption coefficients, nontoxic nature, low cost (abundant elements), and environmental friendliness [1,2]. The thermoelectric properties have been studied only recently, demonstrating high values of the Seebeck coefficient $(S)$ and power factor $\left(S^{2} \sigma\right)$, where $\sigma$ is the electrical conductivity $[3,4]$. Thermoelectric devices can be used for energy harvesting by converting waste heat into electricity and can find applications in electronic refrigeration, for example [5]. The efficiency of a thermoelectric material is characterized by the dimensionless figure of merit $Z T$, where $T$ is the temperature and $Z=$ $S^{2} \sigma / \kappa$, with thermal conductivity $\kappa$ being the sum of electronic $\left(\kappa_{e}\right)$ and lattice $\left(\kappa_{l}\right)$ contributions. A high value of $Z T$ therefore requires high $S^{2} \sigma$ and low $\kappa$. Since both $S$ and $\sigma$ are given by the band structure and vary oppositely under doping [6], good thermoelectric properties are often found in narrow band gap semiconductors with a rather small amount of available charge carriers, such as $\mathrm{CuBiS}_{2}$ [7], $\mathrm{CuGaTe}_{2}$ [4], PbTe [8], and $\mathrm{Bi}_{2} \mathrm{Te}_{3}$ [9].

In general, the behavior of a thermoelectric material is determined by the dominating scattering mechanism [10]. Only few materials exist with $Z T \geqslant 1$ before nanostructuring and/or optimization of the carrier concentration to increase $S^{2} \sigma[11,12]$. Nanostructuring is an effective tool to boost the thermoelectric performance, because $\kappa_{l}$ can be reduced by enhancing the phonon scattering $[13,14]$. For the same reason, two-dimensional semiconductors are promising materials for thermoelectric devices [15-17]. In nanostructured $\mathrm{Bi}_{2} \mathrm{Se}_{3}$, for example, $Z T$ can be enhanced by $40 \%$ as compared to the bulk $[18,19]$, nanocrystalline $\mathrm{Bi}_{0.52} \mathrm{Sb}_{1.48} \mathrm{Te}_{3}$ shows an improvement of more than 50\% [20], and in monolayer SnSe the maximal $Z T$ value as a function of the chemical potential can be several times that of the bulk material [21]. Recent investigations have demonstrated also for other layered compounds that thermoelectric behavior can be strongly modified in a monolayer geometry, for example, in the cases of $\mathrm{MoS}_{2}$ and $\mathrm{WS}_{2}$ [22]. For this reason, in the present paper

\footnotetext{
*udo.schwingenschlogl@kaust.edu.sa
}

we use first-principles calculations along with semiclassical Boltzmann transport theory to compare the electronic and thermoelectric properties of bulk and monolayer $\mathrm{CuSbS}_{2}$ and $\mathrm{CuSbSe}_{2}$ (see Fig. 1).

\section{METHODOLOGY}

First-principles calculations employing the WIEN2k code [23] are performed to optimize the lattice parameters (see the results in Table I) and atomic positions of bulk $\mathrm{CuSbS}_{2}$ and $\mathrm{CuSbSe}_{2}$ (starting from the values given in Refs. [24,25]) until the forces acting on the atoms have decreased to less than $0.01 \mathrm{eV} / \AA$. The obtained $a$ and $b$ lattice parameters are used for building the monolayers, for which the atomic positions are optimized again. The spin orbit coupling can play an important role for the electronic structure in the case of heavy elements and thus is taken into account in our calculations. We use the modified Becke-Johnson (mBJ) method [26], because it is known to provide accurate band gaps for semiconductors $[27,28]$, and include the van der Waals interaction by means of the approach of Ref. [29]. For the Brillouin zone integration in the bulk and monolayer cases, respectively, $7 \times 12 \times 3$ and $13 \times 8 \times 1 k$ meshes are used. The energy cutoff is given by $R_{\mathrm{mt}} K_{\max }=7$ and muffin-tin radii of $2.27 a_{B}, 2.47 a_{B}, 1.85 a_{B}$, and $2.11 a_{B}$ are employed for $\mathrm{Cu}, \mathrm{Sb}, \mathrm{S}$, and $\mathrm{Se}$, respectively. In addition, the wave functions inside the atomic spheres are expanded up to $\ell_{\max }=10$ and the Fourier expansion of the charge density is limited by setting $G_{\max }=12$.

The transport properties are calculated by solving the Boltzmann transport equation within the rigid band (which assumes that the band structure of the host is not altered by doping, and only the chemical potential changes) and constant relaxation time (which assumes that the relaxation time has no energy dependence, which is valid for temperatures up to about $700 \mathrm{~K}$ [4]) approximations, as implemented in the BoltzTraP code [30]. Dense $52 \times 85 \times 22$ (bulk) and $98 \times 62 \times 1$ (monolayer) $k$ meshes are used to obtain accurate carrier group velocities, which determine the transport properties. This approach has been employed successfully in previous studies to screen the potential candidates of thermoelectric materials [31-33]. Using the transport function $\sigma(E)=N(E) v^{2}(E) \tau$, which depends on the density of states $N(E)$, Fermi velocity $v(E)$, 


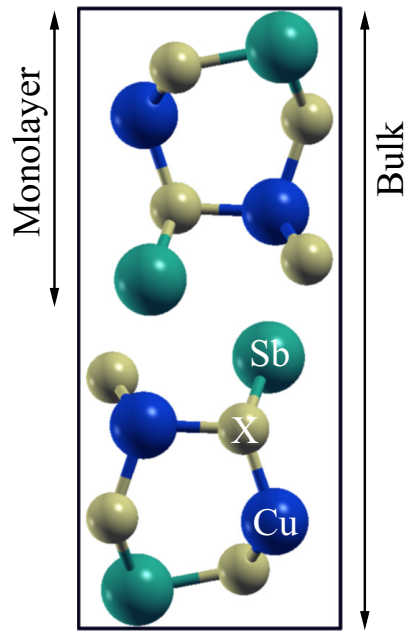

FIG. 1. Crystal structure of bulk and monolayer $\mathrm{CuSb} X_{2}(X=\mathrm{S}$ and $\mathrm{Se})$.
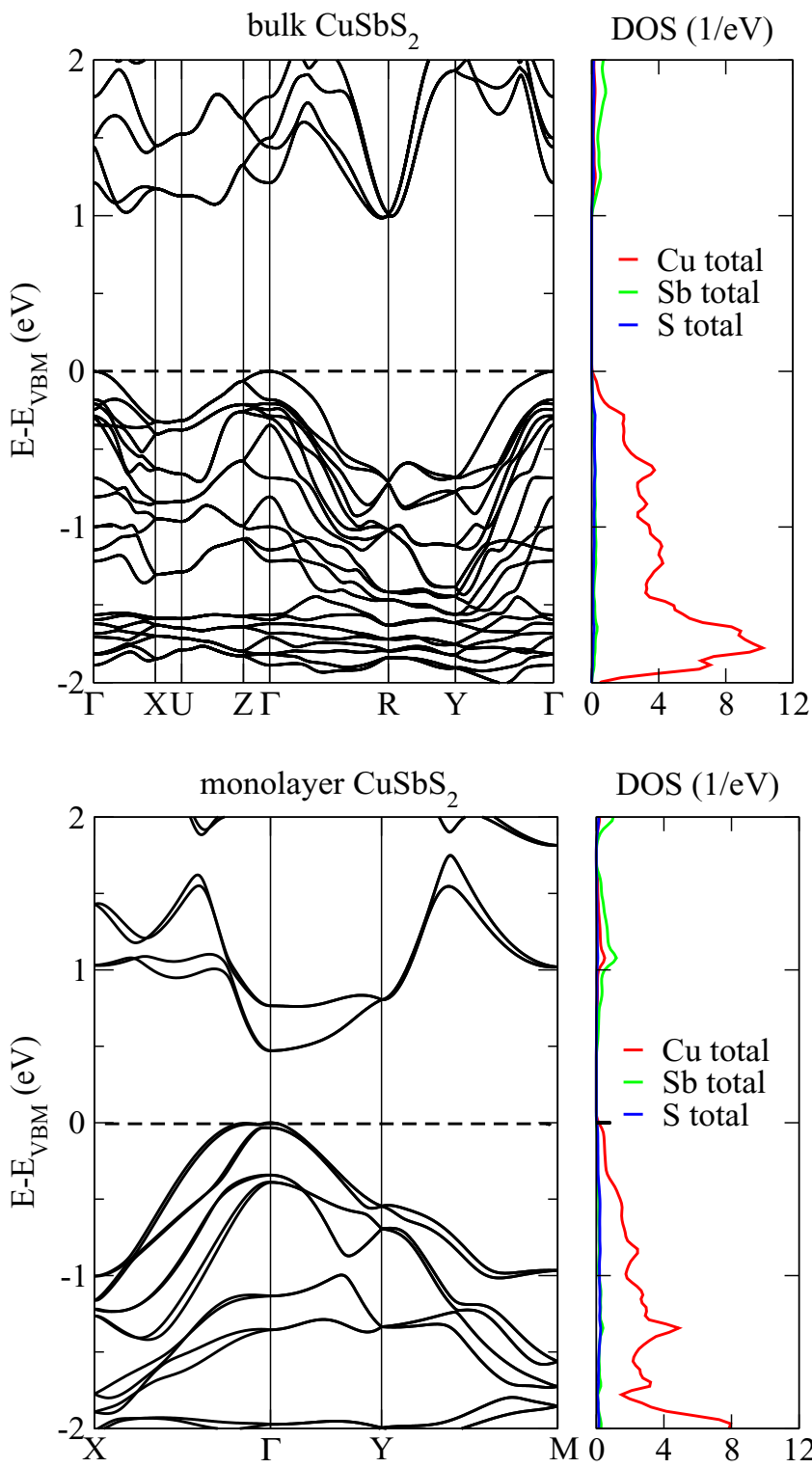

$\operatorname{DOS}(1 / \mathrm{eV})$

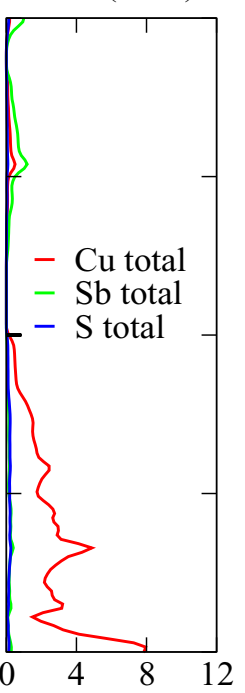

and relaxation time $\tau$, we have

$$
\begin{gathered}
\sigma(T)=-e^{2} \int_{-\infty}^{\infty} d E \sigma(E) \frac{d f(E-\mu, T)}{d E} \\
S(T)=-\frac{e}{T \sigma(T)} \int_{-\infty}^{\infty} d E \sigma(E)(E-\mu) \frac{d f(E-\mu, T)}{d E},
\end{gathered}
$$

and

$$
\kappa_{e}(T)=-\frac{1}{T} \int_{-\infty}^{\infty} d E \sigma(E)(E-\mu)^{2} \frac{d f(E-\mu, T)}{d E},
$$

where $\mu$ is the chemical potential and $f$ is the Fermi function [34].

\section{RESULTS AND DISCUSSION}

Bulk $\mathrm{CuSbS}_{2}$ and $\mathrm{CuSbSe}_{2}$ are orthorhombic with space group Pnma (No. 62) and have four $\mathrm{Cu}$, four $\mathrm{Sb}$, and eight $\mathrm{S} / \mathrm{Se}$ atoms in the unit cell (see Fig. 1). The optimized

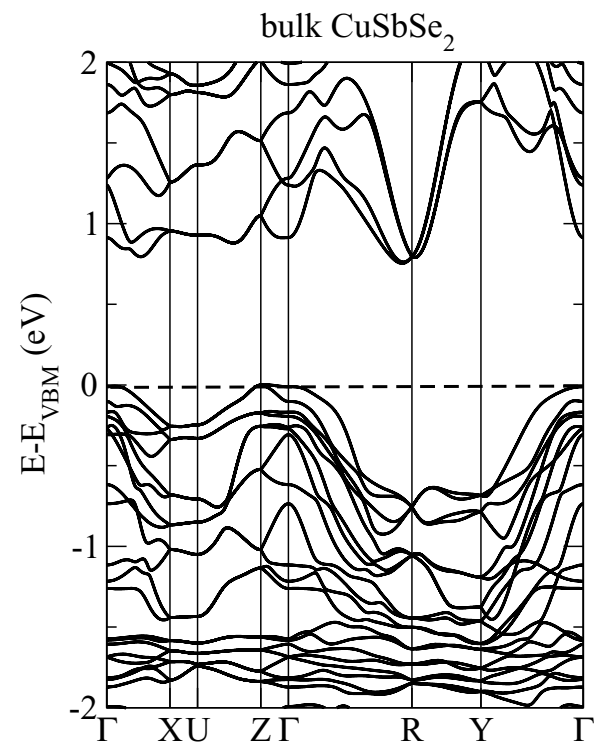

$\operatorname{DOS}(1 / \mathrm{eV})$
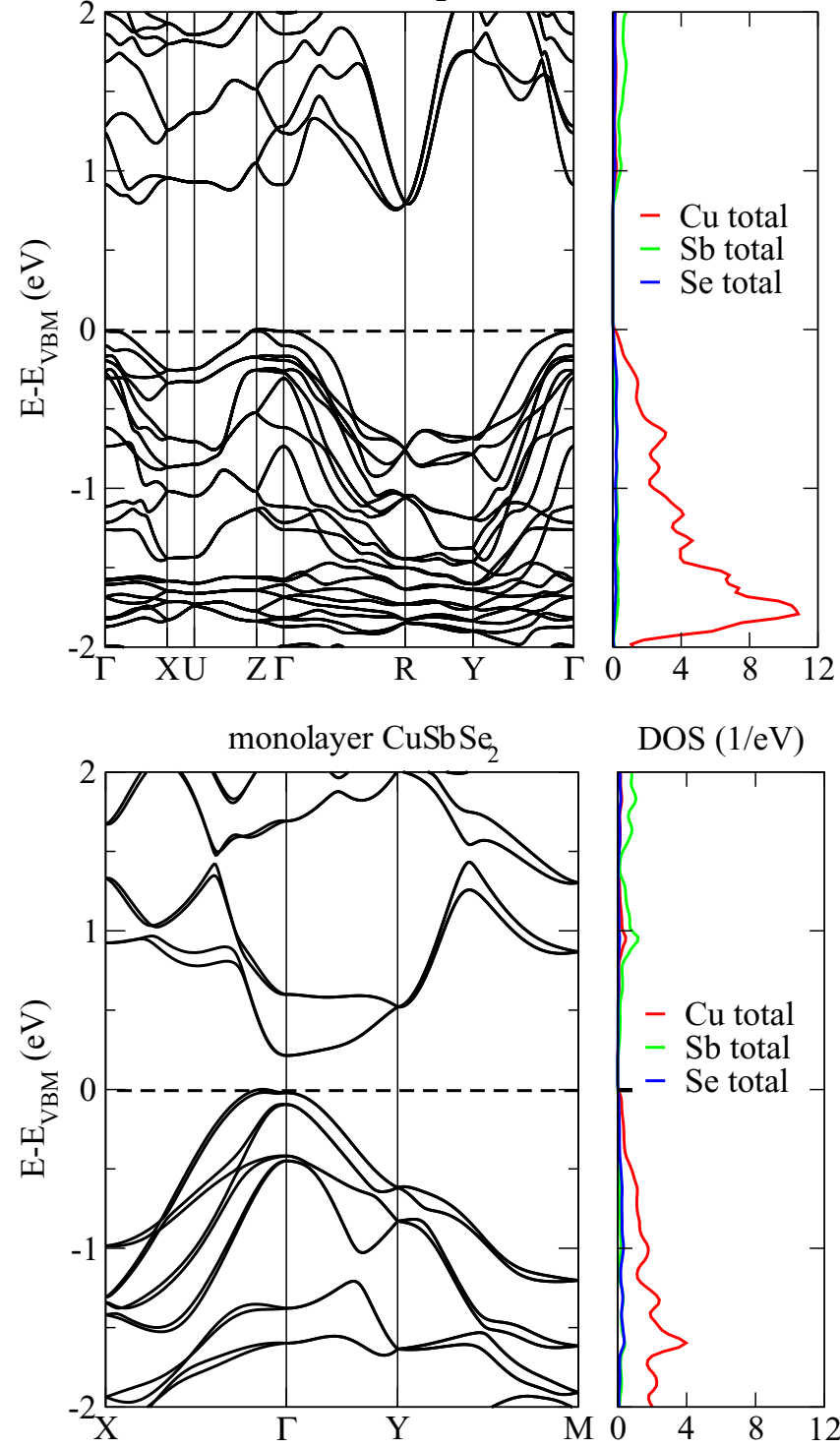

$\operatorname{DOS}(1 / \mathrm{eV})$

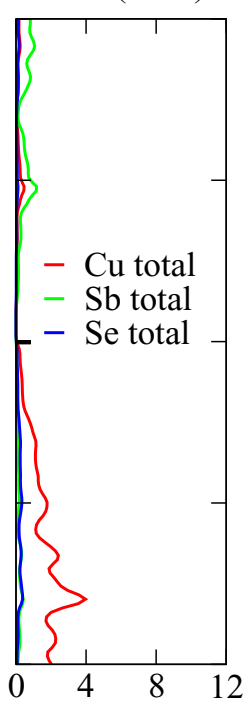

FIG. 2. Band structure and density of states of bulk and monolayer $\mathrm{CuSbS}_{2}$ and $\mathrm{CuSbSe}_{2}$. 
TABLE I. Lattice parameters and band gaps of $\mathrm{CuSbS}_{2}$ and $\mathrm{CuSbSe}_{2}$

\begin{tabular}{lccccc}
\hline \hline & \multicolumn{2}{c}{$\mathrm{CuSbS}_{2}$} & & \multicolumn{2}{c}{$\mathrm{CuSbSe}_{2}$} \\
\cline { 2 - 3 } \cline { 5 - 6 } \cline { 5 - 6 } & Bulk & Monolayer & & Bulk & Monolayer \\
\hline$a(\AA)$ & 6.06 & 6.06 & & 6.50 & 6.50 \\
$b(\AA)$ & 3.82 & 3.82 & & 4.01 & 4.01 \\
$c(\AA)$ & 14.26 & & & 14.87 & \\
$E_{g}(\mathrm{GGA})$ & 0.74 & 0.34 & & 0.49 & 0.15 \\
$E_{g}$ (mBJ) & 1.02 & 0.47 & & 0.78 & 0.21 \\
$E_{g}$ (Expt.) & $1.38[37]$ & & & $1.05[37]$ & \\
\hline \hline
\end{tabular}

distance between the $\mathrm{Sb}$ atoms of neighboring atomic layers is 2.00 and $2.08 \AA$ for $\mathrm{CuSbS}_{2}$ and $\mathrm{CuSbSe}_{2}$, respectively, in reasonable agreement with the experimental values (2.05 and $2.14 \AA$ [35]). The band structures and densities of states in the top row of Fig. 2 show that the valence band is composed mostly of $\mathrm{Cu}$ states and the conduction band of $\mathrm{Sb}$ states. The band gap is found to be indirect, in agreement with the theoretical results in Refs. [4,36] and the experimental results in Refs. [37,38]. The bottom row of Fig. 2 addresses $\mathrm{CuSbS}_{2}$
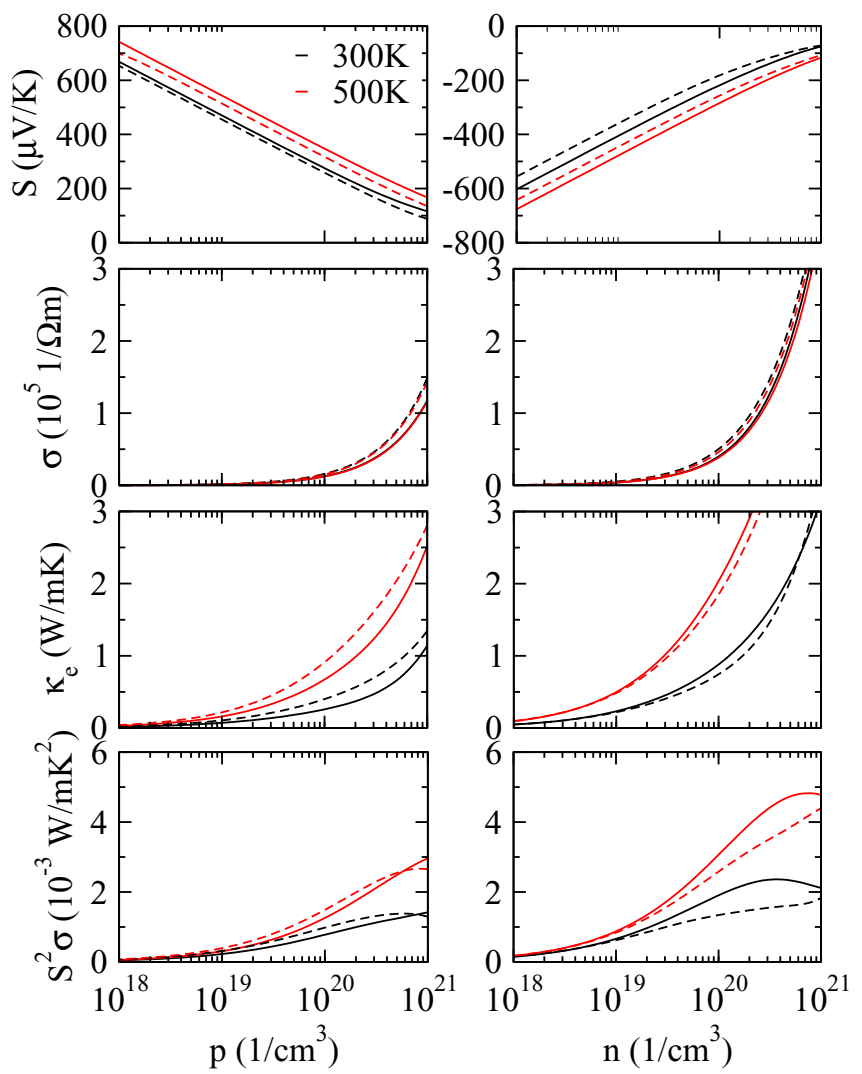

FIG. 3. Seebeck coefficient, electrical conductivity, electronic contribution to the thermal conductivity, and power factor as a function of the hole ( $p$ ) and electron ( $n$ ) densities for bulk $\mathrm{CuSbS}_{2}$ (solid line) and $\mathrm{CuSbSe}_{2}$ (dashed line). and $\mathrm{CuSbSe}_{2}$ monolayers. We find direct $\left(\mathrm{CuSbS}_{2}\right)$ and almost direct $\left(\mathrm{CuSbSe}_{2}\right)$ band gaps at the $\Gamma$ point, which are smaller than those of the bulk compounds, in agreement with Ref. [39] for $\mathrm{CuSbS}_{2}$. This behavior is different from $\mathrm{MoS}_{2}$, for example, where the band gap increases in a monolayer. In $\mathrm{MoS}_{2}$, the $\mathrm{S}$ atoms are located at the van der Waals gap, whereas the local environment of the Mo atoms is the same in the bulk compound and monolayer. As a consequence, the $\mathrm{S}$ dominated valence band edge is shifted to lower energy in the monolayer but not the Mo dominated conduction band edge. In $\mathrm{CuSbS}_{2}$ and $\mathrm{CuSbSe}_{2}$, on the other hand, both $\mathrm{S} / \mathrm{Se}$ and $\mathrm{Sb}$ atoms are located at the van der Waals gap so that a similar effect does not appear. Table I summarizes the calculated and experimental band gaps of bulk and monolayer $\mathrm{CuSbS}_{2}$ and $\mathrm{CuSbSe}_{2}$. The results show that the mBJ method provides much better agreement of the band gaps with experiments than the generalized gradient approximation (GGA) within the Perdew-Burke-Ernzerhof parametrization.

Since the BoltzTraP code calculates the transport coefficients relative to the relaxation time, we determine $\tau=1.1 \times$ $10^{-14} \mathrm{~s}$ for both $\mathrm{CuSbS}_{2}$ and $\mathrm{CuSbSe}_{2}$ from the experimental mobility of $\mu=20 \times 10^{-4} \mathrm{~m}^{2} / \mathrm{V} \mathrm{s}[2,40]$, using $n e^{2} \tau / m=$ $\sigma=n e \mu$. Figure 3 shows the variation of $S$ under hole and electron doping of bulk $\mathrm{CuSbS}_{2}$ and $\mathrm{CuSbSe}_{2}$. The absolute

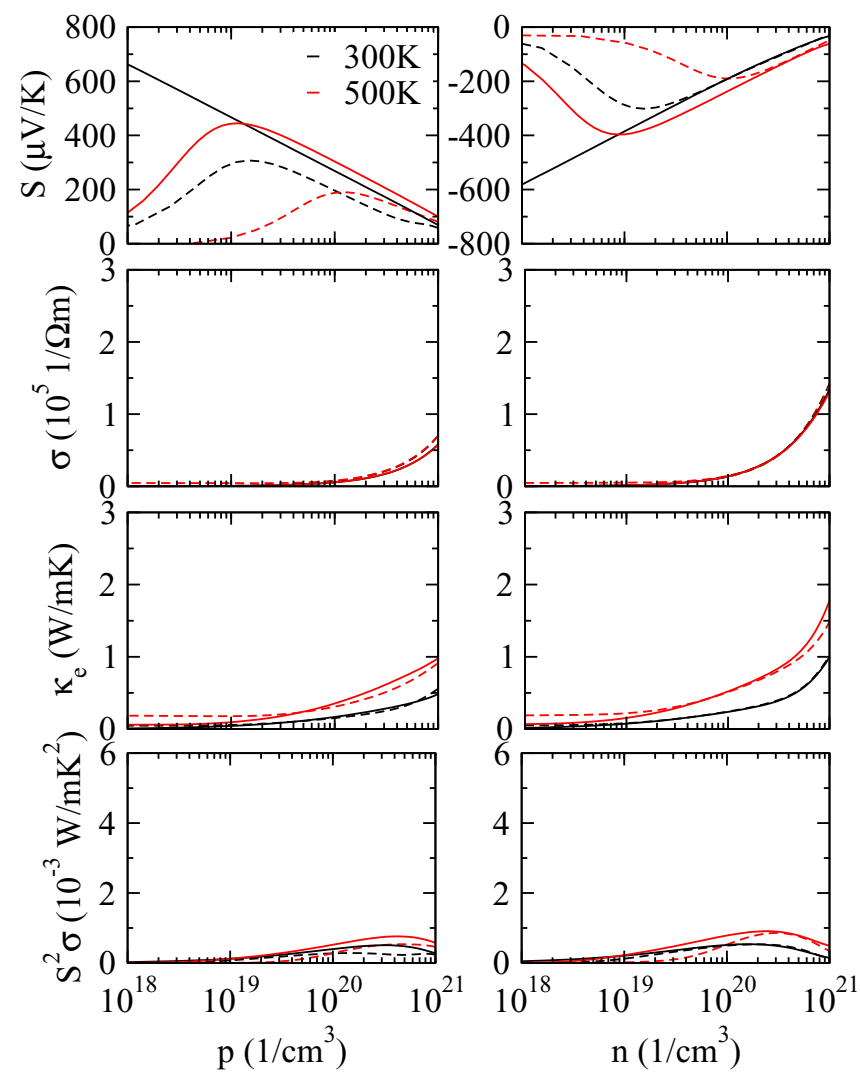

FIG. 4. Seebeck coefficient, electrical conductivity, electronic contribution to the thermal conductivity, and power factor as a function of the hole $(p)$ and electron $(n)$ densities for monolayer $\mathrm{CuSbS}_{2}$ (solid line) and $\mathrm{CuSbSe}_{2}$ (dashed line). 

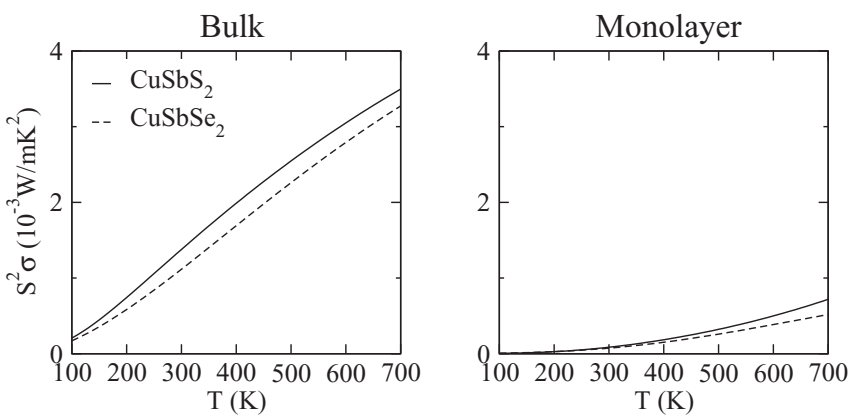

FIG. 5. Power factor of bulk and monolayer $\mathrm{CuSbS}_{2}$ and $\mathrm{CuSbSe} \mathrm{Su}_{2}$ at the hole concentration that maximizes the value at $300 \mathrm{~K}$ in each case.

value of $S$ decreases in both cases with increasing doping and is always higher at $500 \mathrm{~K}$ than at $300 \mathrm{~K}$ by a similar amount, except for high doping. Our results for bulk $\mathrm{CuSbS}_{2}$ are quantitatively similar to those of the calculations reported in Ref. [4], and the value of $S=300 \mu \mathrm{V} / \mathrm{K}$ obtained for bulk $\mathrm{CuSbSe}_{2}$ at $300 \mathrm{~K}$ and a hole doping of $5.2 \times 10^{19} \mathrm{~cm}^{-3}$ agrees well with experiment $(S=375 \mu \mathrm{V} / \mathrm{K})$ [3]. We further observe that $\sigma$ increases in both compounds with the doping (see Fig. 3) being higher for electron than for hole doping and showing almost no temperature dependence. As a result, $S^{2} \sigma$ increases in each case towards a maximum at high doping, with electrons behaving favorably over holes. We note that beyond the constant relaxation time approximation, increasing carrier concentration and temperature will lower $\tau$ and therefore will enhance $S^{2} \sigma$. We have also calculated the thermoelectric properties of bulk and monolayer $\mathrm{CuSbS}_{2}$ by artificially increasing the band gap by $0.3 \mathrm{eV}$ and find only a small effect on the data. Therefore, the exact value of the band gap is not critical for predicting the thermoelectric performance in the range of carrier concentrations under investigation.

Figure 4 shows for monolayer $\mathrm{CuSbS}_{2}$ and $\mathrm{CuSbSe}_{2}$ that the absolute value of $S$ decreases for increasing doping above a certain threshold, being again higher at $500 \mathrm{~K}$ than at $300 \mathrm{~K}$, as expected. The hole and electron densities given in Fig. 4 are obtained from the areal densities of the two-dimensional materials by multiplication with the thickness of the monolayer (5.20 $\AA$ for $\mathrm{CuSbS}_{2}$ and $5.47 \AA$ for $\left.\mathrm{CuSbSe}_{2}\right)$ in order to enable comparison with the bulk results in Fig. 3. There are no experimental data for the monolayers available for comparison. Also in the monolayer case $\sigma$ increases with the doping, showing hardly any difference between $\mathrm{CuSbS}_{2}$ and $\mathrm{CuSbSe}_{2}$ (and being smaller than in the bulk because of modified dispersions at the valence and conduction band edges; see Fig. 2). As a consequence, the trends observed for $S^{2} \sigma$ agree qualitatively with those discussed previously for the bulk compounds. However, the values are reduced because of lower values of $\sigma$. Figure 5 presents $S^{2} \sigma$ as a function of the temperature at the hole concentration that maximizes the value at $300 \mathrm{~K}\left(6 \times 10^{20} \mathrm{~cm}^{-3}\right.$ for bulk and monolayer $\mathrm{CuSbS}_{2}, 5 \times 10^{20} \mathrm{~cm}^{-3}$ for bulk and monolayer $\mathrm{CuSbSe}_{2}$, respectively). We observe that the curves are almost identical for $\mathrm{CuSbS}_{2}$ and $\mathrm{CuSbSe}_{2}$ in the whole temperature range, being significantly lower for the monolayers than for the bulk compounds.

\section{CONCLUSION}

First-principles calculations have been combined with Boltzmann theory to study the electronic and transport properties of bulk and monolayer $\mathrm{CuSbS}_{2}$ and $\mathrm{CuSbSe}$. The electronic band structures turn out to be similar for the two bulk compounds and for the two monolayers. However, the nature of the band gap switches from indirect in the bulk to direct in the monolayer case. Interestingly, the band gap is smaller in the monolayers than in the bulk compounds, because both $\mathrm{S} / \mathrm{Se}$ and $\mathrm{Sb}$ atoms are located at the van der Waals gap of the layered structure so that the valence and conduction band edges are similarly affected by the transition into a two-dimensional geometry. This behavior distinguishes $\mathrm{CuSbS}_{2}$ and $\mathrm{CuSbSe}_{2}$ significantly from other two-dimensional materials, in particular, from the transition metal dichalcogenides. High power factors make $\mathrm{CuSbS}_{2}$ and $\mathrm{CuSbSe}_{2}$ promising materials for thermoelectric applications, the best performance being achieved for hole doping of $6 \times 10^{20} \mathrm{~cm}^{-3}$ for $\mathrm{CuSbS}_{2}$ and $5 \times 10^{20} \mathrm{~cm}^{-3}$ for $\mathrm{CuSbSe}_{2}$.

\section{ACKNOWLEDGMENT}

The research reported in this publication was supported by funding from King Abdullah University of Science and Technology (KAUST).

\section{APPENDIX}

Results of numerical convergence tests are shown in Figs. 6 and 7.

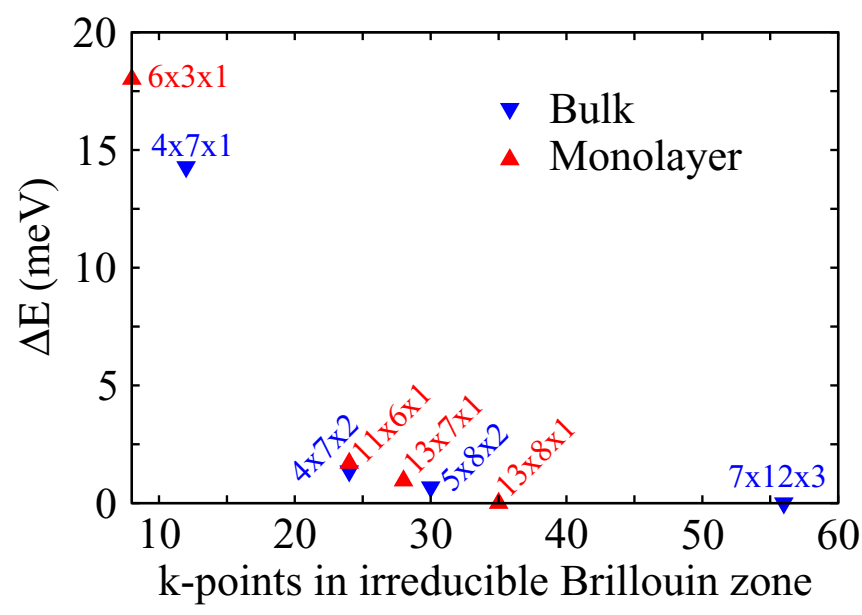

FIG. 6. Total energy convergence for increasing density of the $k$ mesh in the case of $\mathrm{CuSbS}_{2}$. 

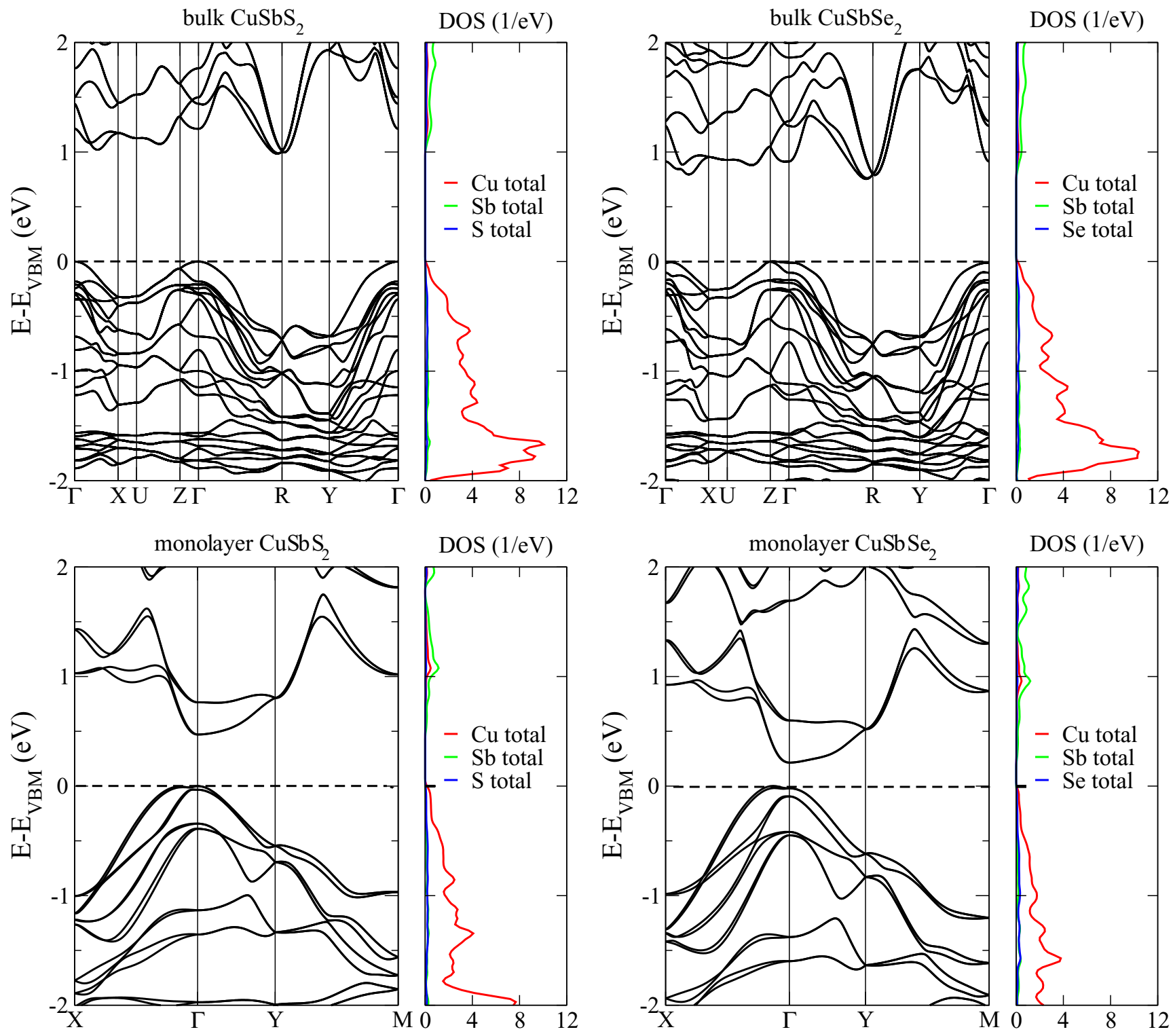

FIG. 7. Recalculation of Fig. 2 with finer $k$ meshes of $8 \times 13 \times 3$ (bulk) and $15 \times 9 \times 1$ (monolayer), demonstrating convergence of the results.

[1] B. Yang, L. Wang, J. Han, Y. Zhou, H. Song, S. Chen, J. Zhong, L. Lv, D. Niu, and J. Tang, $\mathrm{CuSbS}_{2}$ as a promising Earth-abundant photovoltaic absorber material: A combined theoretical and experimental study, Chem. Mater. 26, 3135 (2014).

[2] D.-J. Xue, B. Yang, Z.-K. Yuan, G. Wang, X. Liu, Y.Zhou, L. Hu, D. Pan, S. Chen, and J. Tang, $\mathrm{CuSbSe}_{2}$ as a potential photovoltaic absorber aaterial: Studies from theory to experiment, Adv. Energy Mater. 5, 1501203 (2015).

[3] D. Li and X. Y. Qin, Thermoelectric properties of $\mathrm{CuSbSe}_{2}$ and its doped compounds by $\mathrm{Ti}$ and $\mathrm{Pb}$ at low temperatures from 5 to 310 K, J. Appl. Phys. 100, 023713 (2006).

[4] V. K. Gudelli, V. Kanchana, G. Vaitheeswaran, A. Svane, and N. E. Christensen, Thermoelectric properties of chalcopyrite type $\mathrm{CuGaTe}_{2}$ and chalcostibite $\mathrm{CuSbS}_{2}$, J. Appl. Phys. 114, 223707 (2013).
[5] Y. He, T. Day, T. Zhang, H. Liu, X. Shi, L. Chen, and G. J. Snyder, High thermoelectric performance in non-toxic Earthabundant copper sulfide, Adv. Mater. 26, 3974 (2014).

[6] G. D. Mahan and J. O. Sofo, The best thermoelectric, Proc. Natl. Acad. Sci. USA 93, 7436 (1996).

[7] D. Parker and D. J. Singh, Transport properties of hole-doped $\mathrm{CuBiS}_{2}$, Phys. Rev. B 83, 233206 (2011).

[8] H. Wang, J. Hwang, M. L. Snedaker, I. Kim, C. Kang, J. Kim, G. D. Stucky, J. Bowers, and W. Kim, High thermoelectric performance of a heterogeneous $\mathrm{PbTe}$ nanocomposite, Chem. Mater. 27, 944 (2015).

[9] G. Zhou and D. Wang, Few-quintuple $\mathrm{Bi}_{2} \mathrm{Te}_{3}$ nanofilms as potential thermoelectric materials, Sci. Rep. 5, 8099 (2015).

[10] J. F. Li, W.-S. Liu, L. D. Zhao, and M. Zhou, High-performance nanostructured thermoelectric materials, NPG Asia Mater. 2, 152 (2010). 
[11] T. M. Tritt and M. A. Subramanian, Thermoelectric materials, phenomena, and applications: A bird's eye view, MRS Bull. 31, 188 (2006).

[12] D. Parker and D. J. Singh, Thermoelectric properties of $\mathrm{AgGaTe}_{2}$ and related chalcopyrite structure materials, Phys. Rev. B 85, 125209 (2012).

[13] J. H. Lee, G. A. Galli, and J. C. Grossman, Nanoporous Si as an efficient thermoelectric material, Nano Lett. 8, 3750 (2008).

[14] T. Zhu and E. Ertekin, Phonon transport on two-dimensional graphene/boron nitride superlattices, Phys. Rev. B 90, 195209 (2014).

[15] L. D. Hicks and M. S. Dresselhaus, Effect of quantum-well structures on the thermoelectric figure of merit, Phys. Rev. B 47, 12727 (1993).

[16] L. D. Hicks and M. S. Dresselhaus, Thermoelectric figure of merit of a one-dimensional conductor, Phys. Rev. B 47, 16631 (1993).

[17] T. Zhu and E. Ertekin, Resolving anomalous strain effects on two-dimensional phonon flows: The cases of graphene, boron nitride, and planar superlattices, Phys. Rev. B 91, 205429 (2015).

[18] Y. Saeed, N. Singh, and U. Schwingenschlögl, Thickness and strain effects on the thermoelectric transport in nanostructured $\mathrm{Bi}_{2} \mathrm{Se}_{3}$, Appl. Phys. Lett. 104, 033105 (2014).

[19] L. Cheng, H. Liu, X. Tan, J. Zhang, J. Wei, H. Lv, J. Shi, and $\mathrm{X}$. Tang, Thermoelectric properties of a monolayer bismuth, J. Phys. Chem. C 118, 904 (2014).

[20] W. Xie, X. Tang, Y. Yan, Q. Zhang, and T. M. Tritt, Unique nanostructures and enhanced thermoelectric performance of melt-spun BiSbTe alloys, Appl. Phys. Lett. 94, 102111 (2009).

[21] F. Q. Wang, S. Zhang, J. Yu, and Q. Wang, Thermoelectric properties of single-layered SnSe sheet, Nanoscale 7, 15962 (2015).

[22] S. Kumar and U. Schwingenschlögl, Thermoelectric response of bulk and monolayer $\mathrm{MoSe}_{2}$ and $\mathrm{WSe}_{2}$, Chem. Mater. 27, 1278 (2015).

[23] P. Blaha, K. Schwarz, G. K. H. Madsen, D. Kvasnicka, and J. Luitz, WIEN2k, An Augmented Plane Wave Plus Local Orbitals Program for Calculating Crystal Properties (TU Vienna, Vienna, 2001).

[24] D. Colombara, L. M. Peter, K. D. Rogers, J. D. Painter, and S. Roncallo, Formation of $\mathrm{CuSbS}_{2}$ and $\mathrm{CuSbSe}_{2}$ thin films via chalcogenisation of $\mathrm{Sb}-\mathrm{Cu}$ metal precursors, Thin Solid Films 519, 7438 (2011).

[25] T. Maeda and T. Wada, First-principles study of electronic structure of $\mathrm{CuSbS}_{2}$ and $\mathrm{CuSbSe}_{2}$ photovoltaic semiconductors, Thin Solid Films 582, 401 (2015).
[26] F. Tran and P. Blaha, Accurate Band Gaps of Semiconductors and Insulators with a Semilocal Exchange-Correlation Potential, Phys. Rev. Lett. 102, 226401 (2009).

[27] A. D. Becke and E. R. Johnson, A simple effective potential for exchange, J. Chem. Phys. 124, 221101 (2006).

[28] D. Koller, F. Tran, and P. Blaha, Improving the modified BeckeJohnson exchange potential, Phys. Rev. B 85, 155109 (2012).

[29] S. Grimme, S. Ehrlich, and L. Goerigk, Effect of the damping function in dispersion corrected density functional theory, J. Comput. Chem. 32, 1456 (2011).

[30] G. K. Madsen and D. J. Singh, BoltzTraP. A code for calculating band-structure dependent quantities, Comput. Phys. Commun. 175, 67 (2006).

[31] T. J. Scheidemantel, C. Ambrosch-Draxl, T. Thonhauser, J. V. Badding, and J. O. Sofo, Transport coefficients from firstprinciples calculations, Phys. Rev. B 68, 125210 (2003).

[32] M. S. Diakhate, R. P. Hermann, A. Mochel, I. Sergueev, M. Sondergaard, M. Christensen, and M. J. Verstraete, Thermodynamic, thermoelectric, and magnetic properties of $\mathrm{FeSb}_{2}$ : A combined first-principles and experimental study, Phys. Rev. B 84, 125210 (2011)

[33] S. Azam and A. H. Reshak, Transport properties of $\mathrm{APdCu}\left(\mathrm{Se}_{2}\right)\left(\mathrm{Se}_{3}\right)(\mathrm{A}=\mathrm{K}$ and $\mathrm{Rb})$ : New quaternary copper palladium polyselenides, RSC Adv. 4, 20102 (2014).

[34] P. B. Allen, W. E. Pickett, and H. Krakauer, Anisotropic normalstate transport properties predicted and analyzed for high- $T_{c}$ oxide superconductors, Phys. Rev. B 37, 7482 (1988).

[35] K. Ramasamy, R. K. Gupta, S. Palchoudhury, S. Ivanov, and A. Gupta, Layer-structured copper antimony chalcogenides $\left(\mathrm{CuSbSe}_{x} \mathrm{~S}_{2-x}\right)$ : Stable electrode materials for supercapacitors, Chem. Mater. 27, 379 (2015).

[36] D. J. Temple, A. B. Kehoe, J. P. Allen, G. W. Watson, and D. O. Scanlon, Geometry, electronic structure, and bonding in $\mathrm{CuMCh}_{2}(\mathrm{M}=\mathrm{Sb}, \mathrm{Bi} ; \mathrm{Ch}=\mathrm{S}, \mathrm{Se})$ : Alternative solar cell absorber Materials?, J. Phys. Chem. C 116, 7334 (2012).

[37] J. Zhou, G. Q. Bian, Q. Y. Zhu, Y. Zhang, C. Y. Li, and J. Dai, Solvothermal crystal growth of $\mathrm{CuSbQ}_{2}(\mathrm{Q}=\mathrm{S}, \mathrm{Se})$ and the correlation between macroscopic morphology and microscopic structure, J. Solid State Chem. 182, 259 (2009).

[38] K. Takei, T. Maeda, and T. Wada, Crystallographic and optical properties of $\mathrm{CuSbS}_{2}$ and $\mathrm{CuSb}\left(\mathrm{S}_{1-x} \mathrm{Se}_{x}\right)_{2}$ solid solution, Thin Solid Films 582, 263 (2015).

[39] K. Ramasamy, H. Sims, W. H. Butler, and A. Gupta, Mono-, few-, and multiple layers of copper antimony sulfide $\left(\mathrm{CuSbS}_{2}\right)$ : A ternary layered sulfide, J. Am. Chem. Soc. 136, 1587 (2014).

[40] W. Wubet and D. H. Kuo, Process limitation for p-type $\mathrm{CuSbS}_{2}$ semiconductor with high electrical mobility of $20 \mathrm{~cm}^{2} \mathrm{~V}^{-1} \mathrm{~s}^{-1}$, Mater. Res. Bull. 53, 290 (2014). 